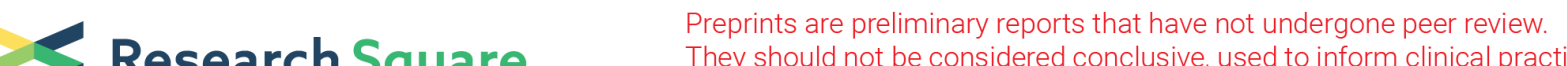 $\begin{array}{ll}\text { Research Square } & \text { They should not be considered conclusive, used to inform clinical practice, } \\ \text { or referenced by the media as validated information. }\end{array}$
}

\section{A thermophilic Cas13 enzyme for sensitive and specific one-pot detection of SARS-CoV-2}

\section{Ahmed Mahas}

King Abdullah University of Science and Technology

\section{Tin Marsic}

King Abdullah University of Science and Technology

Mauricio Masson

King Abdullah University of Science and Technology https://orcid.org/0000-0003-3895-6254

\section{Qiaochu Wang}

King Abdullah University of Science and Technology

\section{Rashid Aman}

King Abdullah University of Science and Technology

\section{Cheng Zheng}

King Abdullah University of Science and Technology

\section{Zahir Ali}

King Abdullah University of Science and Technology

\section{Bernard Ghanem}

King Abdullah University of Science and Technology

MAGDY Mahfouz ( $\square$ magdy.mahfouz@kaust.edu.sa )

King Abdullah University of Science and Technology https://orcid.org/0000-0002-0616-6365

\section{Article}

Keywords: COVID-19, SARS-CoV-2, RT-LAMP, HiT7, RT-LAMP, CRISPR-Cas systems, CRISPR, nucleic acid detection, biosensors, molecular diagnostics, Cas13, Cas13a

Posted Date: September 22nd, 2021

DOl: https://doi.org/10.21203/rs.3.rs-604849/v1

License: (c) (i) This work is licensed under a Creative Commons Attribution 4.0 International License. Read Full License 


\section{Abstract}

Robust, sensitive, and specific diagnostic platforms for early pathogen detection are essential for the identification of infected patients and management of current and future pandemics. CRISPR-Cas systems have been repurposed for SARS-CoV-2 detection in two-pot assays. Two-pot assays require extra steps and are prone to cross-contamination; however, the temperature range of current Cas enzymes limits the development of one-pot assays Here, we characterized TccCas13a, a thermophilic Cas13a enzyme with cis and trans activities from $37-70^{\circ} \mathrm{C}$, and HheCas $13 \mathrm{a}$, which had a limited range and lower activity. We harnessed TccCas13a in a one-pot SARS-CoV-2 assay with two layers of amplification and TccCas13a-mediated collateral degradation of a single-stranded RNA reporter. This assay showed $95 \%$ sensitivity and $100 \%$ specificity compared with RT-qPCR on clinical samples. We also developed a mobile phone application to facilitate data reading, collection, and sharing. Our OPTIMA-dx detection module exhibits key features for point-of-care SARS-CoV-2 screening and pathogen detection in general.

\section{Introduction}

One major constraint on assays is the temperatures at which the enzyme components operate. Indeed, as the discovery of a thermostable DNA polymerase from Thermus aquaticus opened up remarkable possibilities for new assays, the discovery of other enzymes with broader operating temperatures will allow researchers to develop assays that require fewer steps, thus limiting opportunities for contamination and making these easy to use and implement in developing closed system devices for diagnostics.

Currently, there is an urgent need for testing for members of the Coronaviridae family, including Severe acute respiratory syndrome coronavirus 2019 (SARS-CoV-2). RT-qPCR remains the gold standard for SARS-CoV-2 testing, but the need for sophisticated laboratory facilities, and trained personnel, as well as expensive reagents and sample handling, limit its usefulness for frequent testing at massive scales and in resource-limited areas. Advances in synthetic biology and bioengineering have spurred a wave of innovations in molecular diagnostics [1]. One of the most promising detection approaches involves CRISPR/Cas systems. CRISPR-Cas systems have been harnessed in diverse applications, including genome engineering, gene knockdown, virus interference, bioimaging, and diagnostics [2, 3]. Although most applications use Cas endonuclease activity to generate double-stranded DNA breaks, collateral activities of some systems have been harnessed for diagnostic assays. For example, type VI CRISPRCas13 systems possess an RNA-targeted RNA ribonuclease domain; once activated, this domain induces promiscuous collateral degradation of surrounding single-stranded RNA (ssRNA) molecules [4]. Indeed, Cas13 enzymes have been employed for various CRISPR-based detection applications [5-9]. These CRISPR-based modalities have paved the way for developing the next generation of diagnostics by exploiting the highly specific target recognition and cleavage by Cas enzymes, followed by in trans cleavage of reporters by collateral activity [10-13]. The unprecedented versatility, programmability, and 
simplicity of CRISPR-based diagnostics without the need for sophisticated instruments render them amenable for the development of POC diagnostics [14-16].

To increase their sensitivity, CRISPR-based systems have been coupled to isothermal amplification methods such as recombinase polymerase amplification (RPA) or loop-mediated isothermal amplification (LAMP), enabling target nucleic acid detection at attomolar $\left(10^{-18} \mathrm{M}\right)$ levels $[7,9,17]$. Cas13 systems coupled with RT-RPA or RT-LAMP have been employed for SARS-CoV-2 detection with several optimisations to enhance their sensitivity and specificity [18-20]. LAMP isothermal amplification has arisen as the method of choice for sensitive and specific detection, due to its high sensitivity, rapid turnaround time, simple operation, and low cost [21]. In RT-LAMP-coupled CRISPR detection modules, the viral RNA genome is first reverse transcribed, then amplified by LAMP; for detection by Cas13, the amplified DNA is transcribed by RNA polymerase, from T7 priming sites. However, current CRISPR systems use Cas nucleases isolated from mesophilic bacteria and operating best at around $37^{\circ} \mathrm{C}$, while LAMP systems operate at higher temperatures $\left(\sim 55-65^{\circ} \mathrm{C}\right)$. Therefore, RT-RPA- and RT-LAMP-coupled CRISPR detection assays are mostly conducted in two "pots", or tubes, although some studies have used one-pot RPA-CRISPR assays with low sensitivity [20].

Two-pot assays suffer from cross-contamination, as opening the first tube can create aerosols, necessitating the two reactions be conducted in physically separate areas. In addition, recently developed one-pot assays rely on lateral flow readouts that require opening the reaction tube post-amplification, increasing the chance of cross-contamination [22]. Therefore, current work focuses on developing singlepot, single-temperature assays to limit cross-contamination, facilitate end-user handling, enable point-ofcare (POC) applications, and develop at-home testing kits. To provide a simple, reliable one-pot assay, the amplification step (RT-LAMP) and the detection step (CRISPR-Cas enzyme activation and reporter cleavage) should be conducted in a single tube and at a single temperature. In addition, detection platforms for POC use should meet the ASSURED criteria (Accurate, Specific, Sensitive, User-friendly, Rapid, Equipment-free, and Deliverable to end-users), as defined by the World Health Organization [23]. Therefore, additional work to improve assay readouts to facilitate interpretation, accuracy, and data sharing will enhance POC applications. Smartphone-based applications may play key roles in future diagnostic applications.

Identification of thermostable CRISPR-Cas enzymes will enable their simultaneous application with RTLAMP in a single tube. In this work, we report the identification and characterisation of a thermophilic Cas13a orthologue from Thermoclostridium caenicola (TccCas13a) that is highly active at the temperatures required for RT-LAMP. TccCas13a exhibits robust cis and trans catalytic activities at a broad range of temperatures $\left(37-70^{\circ} \mathrm{C}\right)$. We coupled this thermophilic Cas13a variant with RT-LAMP for virus 
detection in a one-pot assay, which we named OPTIMA-dx. We validated our one-pot detection module for SARS-CoV-2 detection in clinical samples from COVID-19 patients. Furthermore, we developed a mobile phone application using a machine learning approach to streamline the reading and collection of test results and allow data sharing with central healthcare systems. These key advances will help in development of an effective and low-cost POC modality for massive-scale testing that detects SARS-CoV2 and future tests for other pathogens.

\section{Results}

\section{Identification of thermophilic Cas13 proteins}

Here, we set out to identify Cas 13 proteins from thermophilic bacteria and test their thermostability at the higher temperatures suitable for one-pot RT-LAMP assays. We interrogated existing Cas13 variants to determine whether they originated from thermophilic hosts. We thus identified HheCas13a from the thermophilic bacterium Herbinix hemicellulosilytica as a potential thermophilic protein [24, 25]. Subsequently, we used the HheCas13a protein sequence as a query in a BLAST-P search against nonredundant protein databases, leading to the isolation of another likely thermophilic Cas13a homologue from Thermoclostridium caenicola (TccCas13a) (Fig. 1a).

We synthesised the TccCas13a gene and used the available clone of HheCas13a for heterologous production in E. coli and purified the recombinant proteins to homogeneity. Subsequently, we conducted differential scanning fluorimetry (DSF) to test their thermostability. Both proteins possessed a denaturation temperature higher than Cas13 enzymes from mesophilic bacteria, e.g., LwaCas13a (Fig. 1b and c). We next reasoned that complexing the crRNA to the HheCas13a and TccCas13a proteins would further stabilise the proteins at higher temperatures. We performed an in silico prediction of the TccCas13a crRNA and used the recently reported crRNA of HheCas13a [25] (Fig. S1). We incubated TccCas13a and HheCas13a with and without their respective in vitro-transcribed crRNAs at different temperatures and separated the complexes by SDS-PAGE. Indeed, TccCas13a and HheCas13a loaded with their cognate crRNAs exhibited higher thermostability, with TccCas13a performing better than HheCas13a (Fig. 1d and 1e). We concluded that both proteins exhibit the needed thermostability for our projected downstream applications requiring cis and trans catalytic activities at higher temperatures.

\section{Characterization of the cis and trans catalytic activities of TccCas13a and HheCas13a}

Our initial characterisation demonstrated that both proteins remained folded at higher temperatures and that loading of the crRNA enhanced their thermostability. We next tested whether their respective ribonucleoprotein (RNP) complexes are active and mediate the cis and trans activities essential for downstream applications. Accordingly, we designed crRNAs targeting a synthetic RNA sequence to 
determine whether either protein exhibited catalytic cis activities at elevated temperatures. Both HheCas13a and TccCas13a proteins did show robust and higher cis catalytic activities at higher temperatures $\left(60^{\circ} \mathrm{C}\right)$ when incubated with their target RNA than when incubated at $37^{\circ} \mathrm{C}$ (Fig. 2a).

Next, we attempted to determine whether HheCas13a and TccCas13a retained non-specific trans degradation activity of ssRNA reporter molecules in the presence of ssRNA target at elevated temperatures. Such collateral activity is critical for diagnostics, as it is the basis for nucleic acid detection, and other applications of CRISPR-Cas13 systems (Fig. 2b). Different Cas13 variants trigger collateral RNase activity with distinct cleavage preferences depending on SSRNA sequences [9]. Therefore, we screened several ssRNA reporters consisting of 6-mers of A or U homopolymers, or a 6-mer probe with a mix of $\mathrm{U}, \mathrm{G}, \mathrm{A}$, and $\mathrm{C}$ nucleotides. TccCas 13 a exhibited robust trans cleavage and a strong preference for the mixed nucleotide reporter. HheCas13a, however, displayed robust trans cleavage using the poly U reporter, consistent with a recent study (Fig. 2c) [25].

Moreover, we compared the trans cleavage activities of HheCas13a and TccCas13a onto ssRNA reporters at $37^{\circ} \mathrm{C}$ and $60^{\circ} \mathrm{C}$ with matching crRNA spacer sequences. While both enzymes performed well at both temperatures, TccCas13a showed a faster and stronger trans cleavage activity at high temperature and low target RNA concentration than HheCas13a (Fig. 2d). Subsequently, we conducted RNA detection assays over a range of temperatures from $37-72^{\circ} \mathrm{C}$ to determine the optimal temperature for TccCas $13 \mathrm{a}$ and HheCas13a. TccCas13a was active over a wide range of temperatures $\left(37-70^{\circ} \mathrm{C}\right)$, while HheCas 13 exhibited robust activity over the more limited temperature range of $37-60^{\circ} \mathrm{C}$ (Fig. 2e). These data provide compelling evidence of the thermostability and robust catalytic activities of TccCas13a and HheCas13a proteins and their usefulness in applications requiring cis and trans catalytic activities.

\section{RT-LAMP-coupled thermophilic CRISPR-Cas13a enzymes for SARS-CoV-2 detection}

Based on the results above, we recognised the possibility of developing a novel and sensitive assay by coupling RT-LAMP isothermal amplification with in vitro transcription and subsequent Cas13-based detection in one-step at the same temperature by using HheCas13a and/or TccCas13a. Two rounds of amplification of the target virus or genome can be achieved: the first round via RT-LAMP isothermal amplification; the second via the in vitro transcription of the RT-LAMP products with T7 RNA polymerase. We first tested the applicability of this modality using TccCas13a and HheCas13a in a two-pot RT-LAMP assay. To ensure sensitive detection, pre-amplifying the RNA target of interest is a necessary step [7]. We chose RT-LAMP isothermal amplification and used well-established primer sets from previous reports to target and amplify conserved regions in the SARS-CoV-2 $N$ gene, named here STOPCovid primers (SC) [22]. However, because Cas13 proteins target RNA, we modified these primers by appending a T7 
promoter sequence to the $5^{\prime}$ end of the first half of either the forward inner primer (FIP) or the backward inner primer (BIP) (Fig. 3a). Therefore, during LAMP amplification, the T7 promoter sequence should get incorporated into the amplified DNA products, providing a suitable template for the T7 RNA polymerase to transcribe the amplified LAMP product in vitro and generate RNA targets for Cas13 detection (Fig. 3b).

We designed several crRNAs for both TccCas13a and HheCas13a targeting a highly conserved region in the SARS-CoV-2 N-gene. We first showed that both HheCas13a and TccCas13a exhibit robust activity in RT-LAMP buffer (isothermal buffer) (Fig. S2). We then performed an initial screening of these crRNAs and primers in two-pots settings, as described above. Most of the tested crRNAs showed robust performance and high detection signal, with few crRNAs showing no activity (Fig. S3). These results supported the strong detection of RT-LAMP products when using crRNAs, confirming that 1) the amplification of the synthetic SARS-CoV-2 genome is successful with the modified primers; 2) the T7 promoter is successfully integrated into the amplified products; 3) T7 RNA polymerase can use the amplified amplicons to generate Cas13a substrates that activate Cas13a enzymes to degrade ssRNA reporters and generate signal output (Fig. 3b, Fig. S3).

\section{Establishment and optimization of a one-pot RT-LAMP with TccCas13a for SARS-CoV-2 detection}

Both thermophilic Cas13a enzymes exhibited a practical and robust catalytic activity at higher temperatures in a two-pot assay, which motivated us to capitalise on their thermostability to establish a one-pot RT-LAMP-coupled CRISPR assay for the detection of SARS-CoV-2. We first investigated if target detection in a one-pot assay would be feasible at a single temperature. To this end, we rescreened all crRNAs and primer sets in one-pot settings, leading to the identification of a combination of crRNA and primer set with the most specific and efficient detection of the SARS-CoV-2 RNA, namely TccCas13a crRNA\#13 when used with the T7-FIP modified primer set (Fig. 3c). Interestingly, although HheCas13a exhibited strong detection signal in two-pot settings, we observed no significant detection signal in onepot settings with any crRNA. By contrast, TccCas13a was consistent in specifically and efficiently detecting SARS-CoV-2 target in one-pot with the optimised combination of primers and crRNA (Fig. S4). Therefore, we selected TccCas13a for further optimisation and development of one-pot SARS-CoV-2 detection.

We set out to further optimise all reagents to improve the performance, sensitivity, and specificity of the assay for SARS-CoV-2 detection. We first proceeded to optimise the reaction chemistry by testing several Bst DNA polymerases. We noticed that optimal sensitivity and efficiency of our one-pot assay could only be achieved with certain commercially available Bst DNA polymerases (Fig. 3d), which might be a reflection of different buffer compositions, and particularly salt concentrations that would negatively 
affect Hi-T7 RNA polymerase activity. We further optimised and improved the reaction performance by titrating the Bst DNA polymerase, Hi-T7 RNA polymerase, $\mathrm{Mg}^{2+}$ and TccCas13a RNP concentrations in the reaction (Fig. S5).

Because different biochemical reactions perform optimally at different temperatures in our one-pot assay, we tested the performance of the one-pot assay at different temperatures. The optimal temperature for the one-pot detection assay was $56^{\circ} \mathrm{C}$, with diminished performance at higher or lower temperatures, probably due to the reduced performance of the LAMP amplification at lower temperatures, and of the HiT7 RNA polymerase at higher temperatures (Fig. 3e).

\section{Evaluation of OPTIMA-dx assay for SARS-CoV-2 detection and clinical validation.}

To enable large-scale screening during a pandemic, performing diagnostic assays at POC or outside of laboratory settings is critical. Since the use of sophisticated fluorescence detection instruments such as qPCR machines or plate readers complicate the achievement of such goal, CRISPR diagnostic approaches have adapted lateral flow detection in an effort to develop a simple visual readout that can expedite accurate diagnostics at POC settings [9]. However, despite the efficiency and simplicity of such approach, the reaction tubes need to be opened for subsequent lateral flow detection readouts, thus increasing the chance of aerosols and cross-contamination. As an alternative, we sought to couple our assay with a portable device. Using a modified RNA reporter molecule conjugated to a 5 ' HEX fluorescent molecule instead of FAM, TccCas13a collateral cleavage of such a reporter produced a bright signal visible with a hand-held, inexpensive fluorescence visualiser (P51 Molecular Fluorescence Viewer), allowing simple visualisation and interpretation of the results (Fig. 4a). We termed this one-pot assay with visual detection OPTIMA-dx (One-pot thermophilic Cas13 and isothermal amplification module for nucleic acid detection).

With these optimised reaction conditions, we next evaluated the analytical limit of detection (LoD) of OPTIMA-dx using synthetic SARS-CoV-2 RNA as input. We estimated the LoD of OPTIMA-dx assay to be 10 copies $(\mathrm{cp}) / \mu \mathrm{L}$, an improved sensitivity relative to other reported Cas13-based one pot assays for SARS-CoV-2 detection (Fig. 4b) [20]. To test specificity and absence of cross-reactivity, we challenged OPTIMA-dx with other common human viruses, including SARS-CoV-1, MERS, H1N1, HCoV-OC43, HCoV229E, and HCoV-NL63. OPTIMA-dx showed high specificity to SARS-CoV-2, with no cross-reactivity against any of the other tested viruses (Fig. 4c). We next assessed how storage at common storage temperatures influenced the performance of a pre-assembled OPTIMA-dx master mix. Although the OPTIMA-dx reaction did lose activity after storage for $48 \mathrm{~h}$ at $4^{\circ} \mathrm{C}$, the detection reaction remained active when stored at $-20^{\circ} \mathrm{C}$ for $48 \mathrm{~h}$ (Fig. $4 \mathrm{~d}$ ). 
To ensure reliability of SARS-CoV-2 detection kits, the Federal Drug Administration (FDA) guidelines (Catalog \# 2019-nCoVEUA-01, CDC, 2019) emphasise the importance of including a positive sample, or internal control, as an indicator of proper sample handling, RNA extraction, template quality and integrity, and validity of reagents. In particular, a negative SARS-CoV-2 readout should be considered invalid if the internal control is negative as well. We thus tested the human ribonuclease $P($ RNase $P)$ transcripts as internal control for OPTIMA-dx SARS-CoV-2 detection assay, whereby each sample can be evaluated by two OPTIMA-dx reactions for the detection of SARS-CoV-2 and the RNase $P$ internal control (Fig. 4e). Accordingly, we designed two crRNAs targeting a region of RNase $P$ amplified with RT-LAMP primers developed in previous reports that we modified with the T7 promoter sequence appended to the FIP primer [26]. Both crRNAs showed efficient and specific detection, with crRNA 1 showing a faster detection signal compared to crRNA 2, prompting us to select crRNA 1 for the OPTIMA-dx RNase Passay (Fig. 4f).

Next, we sought to validate OPTIMA-dx with total RNA extracted from oropharyngeal or nasopharyngeal swab samples collected from suspected SARS-CoV-2 patients. After RNA extraction following the protocol approved by the Centers for Disease Control Emergency Use Authorization (CDC EUA), we identified the samples positive for SARS-CoV-2 by RT-qPCR. We then conducted our validation assays of OPTIMA-dx using RNA extracted from 45 randomized samples, 40 positive samples with $\mathrm{Ct}$ values ranging between 14-34, and 5 negative samples (Fig. 5a). We detected a positive OPTIMA-dx signal with all tested samples, with the exception of the negative samples and no template control (NTC) within $1 \mathrm{~h}$. However, we will note that samples with Ct values above 30 show a weaker signal compared to samples with $\mathrm{Ct}$ values below 30 (Fig. 5a). These results indicated that OPTIMA-dx can reliably detect SARS-CoV-2 in patient samples within $1 \mathrm{~h}$, with a simple visual readout for $\mathrm{Ct}$ values up to 34 .

\section{Development of a mobile phone application to collect and share SARS-CoV-2 test results}

Fluorescence detection devices like any plate reader or real-time PCR machines are choice devices to measure any end-point or real-time signal in a sample. However, at a POC settings with fewer resources and no specialised training, smartphone-based imaging is becoming popular in biomedical applications for easy data accessibility and sharing. To facilitate data collection and sharing of SARS-CoV-2 test results as well as interpretation of the OPTIMA-dx readout, we developed a deep learning-based approach to design and develop a mobile phone application capable of collecting and reading OPTIMA-dx results from the low-cost P51 Molecular Fluorescence Viewer at POC settings (Fig. 5b). The dataset of fluorescent images used for training the software consisted of many random images annotated manually as positive or negative to set the proper fluorescence intensity threshold. The software was then trained and tested multiple times to reach the best mAP (mean average precision) value with this dataset. The 
application allows the user to easily take a picture of PCR strips or upload an already captured image of a PCR strip illuminated by a transilluminator. The software then determines the location of each tube, calculates a probability score for each target category and classifies each tube as positive (green bounding box) or negative (red bounding box) samples based on the intensity of the fluorescent signal (Fig. 5c). The entire image processing, from capturing the reaction tubes to the final app output results, takes less than $1 \mathrm{~min}$. Once the image is captured, it can be uploaded and processed by the software (Supplementary note 2).

We validated the ability of the OPTIMA-dx smartphone application to identify and call positive and negative readouts from OPTIMA-dx results: the app correctly determined the fluorescence status of each sample with good accuracy (Fig. S6). We next evaluated the app on the 45 clinical samples tested with OPTIMA-dx: the app identified 38 out of the 45 samples tested as positive. Notably, the two SARS-CoV-2 positive samples deemed negative by the app had the highest Ct values (31 and 34) and thus lowest intense fluorescent signal of all samples (Fig. S7). We also ran OPTIMA-dx for $R N a s e ~ P$ in the same samples, resulting in 43 samples testing positive for $R$ Nase $P$ out of the 45 samples tested, using the OPTIMA-dx one-pot assay and app (Fig. S8). We conclude that OPTIMA-dx can reach a performance of $95 \%$ sensitivity and $100 \%$ specificity in patient samples when combined with the mobile app, exhibiting high concordance with RT-qPCR data (Fig. 5d).

\section{Discussion}

In this study, we provide: 1 ) the first identification and characterization of thermophilic Cas13a enzymes, thereby expanding the molecular engineering toolbox of CRISPR systems for RNA substrates; 2) the development of a one-pot RT-LAMP assay for specific and sensitive detection of SARS-CoV-2 to facilitate application at POC sites and limit cross-contamination; 3 ) the development and use of mobile phone application coupled with the portable, affordable P51 detection to collect and share testing data with central facilities.

Since their discovery, Type VI CRISPR Cas13 systems have provided efficient and versatile tools for RNA manipulation [27, 28]. However, all Cas13 work to date has been restricted to temperatures around or below $42^{\circ} \mathrm{C}$. Mining datasets from diverse natural contexts, including thermophiles, helped us uncover novel thermophilic variants that evolved naturally to provide immunity to their hosts. Indeed, we showed that HheCas13a and TccCas13a are thermostable enzymes that are active at elevated temperatures, with TccCas 13 a being active over a wider temperature range $\left(37-70^{\circ} \mathrm{C}\right)$. These thermophilic Cas $13 \mathrm{a}$ enzymes will open diverse biotechnological applications for RNA-guided Cas13a ribonucleases at a broad temperature range and under harsh experimental or environmental conditions, especially if complexed with their corresponding crRNAs. For example, Cas13 has recently been gaining interest for therapeutics and disease research, including cancer gene therapy and antiviral therapeutics [29-32]. However, in vivo protein stability is critical for successful application [33] and some proteins, such as LwaCas13a, 
mediate efficient knockdown only when fused to a stabilizing domain [34]. Notably, thermostabilisation of proteins results in better stability in vivo $[35,36]$. Therefore, the robust activity and thermostability of TccCas13a make this protein a promising candidate for further structural studies and potential in vivo RNA targeting applications. Moreover, thermostable enzymes (including thermostable Cas9) have enabled important genome editing applications in thermophiles [37]; specific RNA targeting at elevated temperatures beyond the range of previously reported Cas13 proteins is key to creating new tools for use in industrially important thermophiles, for which no CRISPR-Cas13 system has been reported.

To boost sensitivity, CRISPR-based diagnostics typically involve an amplification step, preferably by isothermal amplification. Our detection module employs RT-LAMP to generate a massive number of amplicons with a second amplification step, achieved with thermostable T7 RNA polymerase, to generate RNA transcripts that can be detected by thermophilic Cas13a. Although RT-RPA amplification was the preferred method for SHERLOCK and other CRISPR-dx modules, severe limitation in RPA reagent supply was observed during the COVID-19 pandemic. By contrast, LAMP reagents are readily available from different suppliers and can be produced in-house [38]. Therefore, LAMP was adapted to develop sensitive CRISPR diagnostics for SARS-CoV-2 detection, including DETECTR [17], iSCAN [38], DISCoVER (Cas13based module) [18] and the first and only FDA-authorized CRISPR-Cas13 based diagnostics test (SHERLOCK CRISPR SARS-CoV-2 Kit, IDT). However, all the above diagnostic methods are performed in two-pot settings, as their Cas enzymes function at around $37^{\circ} \mathrm{C}$ and therefore cannot tolerate the high temperatures needed for LAMP $\left(\sim 55-65^{\circ} \mathrm{C}\right)$. Such two-pot settings are not ideal for POC settings due to the increased chance of cross-contamination, which can be overcome with the implementation of thermophilic DNA-targeting Cas12 proteins $[22,39]$. Interestingly, recent reports have turned to RNAtargeting CRISPR-Cas systems from class I type I and type III for SARS-CoV-2 diagnostics. Their thermophilic nature is an appealing feature for coupling with RT-LAMP to develop one-pot detection assays. However, both systems have low sensitivity in one-pot settings with RT-LMAP, forcing their use in two-pot settings. In addition, the required class I complexes are large molecules, complicating their purification and use in vitro $[40,41]$. Therefore, our thermophilic Cas13a protein offers a great advance in diagnostics and will enable the development of other applications.

Our OPTIMA-dx detection module has several other advantages that make it suited for POC applications. Most CRISPR-dx assays developed for SARS-CoV-2 detection do not include a human internal control, which would ensure test reliability, especially in POC settings. We successfully adapted our detection system to the human RNase $P$ transcript and we expect that OPTIMA-dx will have a broad range of applications for pathogen detection. In addition, we demonstrated that OPTIMA-dx reagents can tolerate storage at $-20^{\circ} \mathrm{C}$ for at least $48 \mathrm{~h}$, which would facilitate pre-assembly of OPTIMA-dx reactions for largescale screening in POC settings. Moreover, our OPTIMA-dx detection module may not require RNA extraction and should be compatible with direct lysis methods, including chemical reduction and ion 
chelation. Finally, we integrated our detection module with the portable P51 Molecular Fluorescence Viewer to facilitate sample reading using the HEX reporter and developed a machine learning module for efficient data collection and sharing of the test results. The P51 device allows the processing of eight samples simultaneously, and the corresponding photograph can be saved with the positive and negative labels on the image. The P51 device can be adapted to accommodate a higher number of samples. The results of the analysis can also be saved in a readable format and shared with the patients or concerned health organizations on demand.

The use of mobile phones for data collection and sharing may be instrumental in contact tracing and tracking, as it streamlines data sharing. The portability and ease of using a smartphone-based detection device may help untrained personnel interpret results from samples with weak signals. Importantly, further training of the app with more samples will be needed to improve its performance in POC settings. Overall, the software provides an additional diagnosis validation and enables fast data sharing, making the entire diagnostic process affordable and accessible to a larger section of society.

In conclusion, we characterised a novel thermophilic Cas13a variant and developed a one-pot RT-LAMPcoupled CRISPR-Cas13a assay for sensitive and specific SARS-CoV-2 detection. Our work provides a viable platform for COVID-19 detection in limited-resource settings. We envision that our current detection module will be used to build a device for at-home or POC testing for COVID-19 and other pathogens. Moreover, the thermophilic Cas13a variants reported in this work will have other applications beyond diagnostics, including in RNA knockdown, editing, imaging, and virus interference. This work thus expands the applications of CRISPR-Cas 13 systems and offers new possibilities for transcriptome engineering and diagnostics at higher temperatures.

\section{Materials And Methods}

\section{Computational identification of a thermophilic CRISPR/Cas13a}

We manually interrogated various existing Cas13 enzymes and their bacterial hosts to identify potential thermophilic Cas13s originating from thermophilic organisms. After the identification of HheCas13a as a potential thermophilic Cas13 protein, we used its protein sequence as query for BLAST analysis against the NCBI non-redundant (nr) protein database using default settings. Only sequences with query coverage above $80 \%$ were considered for a second round of host interrogation (focused on growth conditions using the BacDive database and other resources). We identified TccCas13a (accession \# WP_149678719.1) from Thermoclostridium caenicola (strain DSM 19027) as another potential thermophilic Cas13 protein. 
A phylogenetic tree was reconstructed using protein sequences of different Cas13 proteins belonging to different families/subtypes of Class II/type VI CRISPR-Cas systems. All protein sequences were organized in a single .txt file and aligned using MUSCLE in MEGAX software with default settings. The phylogenetic reconstruction was based on the maximum-likelihood method with the WAG+G+F model and 1,000 bootstrap samplings. The generated output file (.nwk) was visualized using TreeGraph_2.

The T. caenicola genome (GenBank\# NZ_FQZP01000023.1) was submitted to the CRISPRCasFinder program [42] to identify the associated CRISPR array. CRISPRDetect [43] was then used to predict the orientation of the direct repeat in the TccCas13a CRISPR array.

\section{Cas13 protein production and purification}

The expression vector pC013-Twinstrep-SUMO-huLwCas13a_WT for the production of LwaCas13a was obtained from Addgene (plasmid \#90097); the purification of recombinant LwaCas13a was performed following a previously published protocol [44] .The expression vector P2CT-His-MBP-Hhe_Cas13a_WT for the production of HheCas13a was obtained from Addgene (plasmid \#91871) and the purification of the recombinant HheCas13a was performed following a previously published protocol [25]. To generate the plasmid for TccCas13a production and purification, the TccCas13a coding sequence codon-optimized for E. coli was synthesized (GenScript) de novo and subcloned in-frame downstream of the sequences encoding the His and SUMO tags into the His6-TwinStrep-SUMO bacterial expression vector (Addgene \#90097) by replacing the LwaCas13a encoding sequence with TccCas13a sequence using the BamHI and Notl restriction sites. Purification of the TccCas13a protein was performed following the protocol of Kellner et al. (2019) [44] with a few modifications. Briefly, the TccCas13a expression vector was transformed into the $E$. colis strain BL21. Starter cultures were prepared by growing single colonies in LB broth containing $100 \mu \mathrm{g} / \mathrm{mL}$ ampicillin for about $12 \mathrm{~h}$ at $37^{\circ} \mathrm{C}$. Next, $25 \mathrm{~mL}$ of starter culture was used to inoculate $1 \mathrm{~L}$ of Terrific Broth (IBI Scientific) containing $100 \mu \mathrm{g} / \mathrm{mL}$ ampicillin, and the $1-\mathrm{L}$ cultures (4 L total) were incubated at $37^{\circ} \mathrm{C}$ until an $\mathrm{OD}_{600}$ of $\sim 0.5$. Cells were then placed at $4^{\circ} \mathrm{C}$ for $30 \mathrm{~min}$, and protein production induced with the addition of $0.5 \mathrm{mM}$ IPTG (isopropyl ß-D-1-thiogalactopyranoside). Cultures were then incubated overnight at $16^{\circ} \mathrm{C}$ with shaking at $180 \mathrm{rpm}$. Next, cells were harvested by centrifugation for $20 \mathrm{~min}$ at $4^{\circ} \mathrm{C}$ at 4,000 rpm. Cell pellets were resuspended in lysis buffer $(50 \mathrm{mM}$ Tris$\mathrm{HCl} \mathrm{pH} \mathrm{7.5,} 300 \mathrm{mM} \mathrm{NaCl}, 5 \%$ glycerol, $1 \mathrm{mM}$ TCEP [tris(2-carboxyethyl)phosphine], $4.5 \mathrm{mM} \mathrm{MgCl}$, $1 \mathrm{mM}$ PMSF, EDTA-free protease inhibitor [Roche]) and with $1 \mathrm{mg} / \mathrm{mL}$ lysozyme (L6876, Sigma). Cells were lysed by sonication and clarified by centrifugation at $12,000 \mathrm{rpm}$ for $60 \mathrm{~min}$ at $4{ }^{\circ} \mathrm{C}$. The soluble $6 \mathrm{xHis-}$ SUMO-TccCas13a protein was then purified from cleared lysate with an affinity chromatography column (HiTrap Q HP, $5 \mathrm{~mL}$ GE Healthcare) (AKTA PURE, GE Healthcare) followed by concurrent removal of the 6xHis-SUMO tag by digestion with the SUMO protease and overnight dialysis in dialysis buffer $(50 \mathrm{mM}$ Tris-HCl pH 7.5, $200 \mathrm{mM} \mathrm{KCL}, 5 \%$ glycerol, $1 \mathrm{mM}$ TCEP). The cleaved protein was concentrated to $1.5 \mathrm{~mL}$ by Amicon Ultra-15 Centrifugal Filter Units (100 kDa NMWL, UFC905024, Millipore) and further purified via size-exclusion chromatography on a S200 column (GE Healthcare) in gel filtration buffer ( $50 \mathrm{mM}$ Tris$\mathrm{HCl}, 200 \mathrm{mM} \mathrm{KCL}, 10 \%$ glycerol, $1 \mathrm{mM} \mathrm{TCEP}, \mathrm{pH}$ 7.5). The protein-containing fractions resulting from the gel filtration were pooled, snap-frozen, and stored at $-80^{\circ} \mathrm{C}$. 


\section{Nucleic acid preparation}

A short region of the SARS-CoV-2 $\mathrm{N}$ gene sequence was used as the target sequence in all preliminary characterization and optimization experiments of thermophilic Cas13 to screen collateral reporters and assess thermostability of Cas13 proteins. The $N$ gene target RNA sequence was prepared by in vitro transcription of PCR amplicons containing the T7 promoter sequence using the 2019-nCoV_N_Positive Control plasmid (10006625, IDT) as PCR template. PCR amplicons were purified (QIAquick PCR Purification Kit, QIAGEN) and transcribed in vitro using the HiScribe T7 Quick High Yield RNA Synthesis Kit (E2050, NEB). The transcripts were then purified with Direct-zol RNA Miniprep Kits (R2050, Zymo Research) following the manufacturer's instructions, and the purified RNA was stored at $-80^{\circ} \mathrm{C}$.

HheCas13a and TccCas13a crRNAs were designed to target the sequence of the Ngene in the SARS-CoV2 genome. For crRNA production, templates for in vitro transcription were generated using single-stranded DNA oligos (IDT) containing a T7 promoter, scaffold, and spacer in reverse complement orientation, and were then annealed to the T7 forward primer in Taq DNA polymerase buffer (Invitrogen). The annealed oligos were then used as templates for subsequent in vitro transcription as described above.

To establish the thermophilic Cas13-based one-pot assay, control synthetic SARS-CoV-2 viral genomic sequences were ordered as synthetic RNA (Twist Bioscience, 102024), diluted to 10,000 RNA copies/ $\mu \mathrm{L}$ and used at the indicated concentrations.

For RT-LAMP amplification (described below), previously published LAMP primers designed to amplify the SARS-CoV-2 Ngene [22] were used, with the following modifications. The FIP or BIP primers were designed with the T7 promoter sequence appended at the $5^{\prime}$ end of the first half of the primers.

\section{Differential scanning fluorimetry (DSF)}

DSF was performed using 5 to $15 \mu \mathrm{M}$ of purified Cas 13 protein in gel filtration buffer (with $5 \%$ glycerol) containing $10 \%$ SYPRO Orange fluorescent dye (S6650, ThermoFisher) in a final reaction volume of 35 $\mu \mathrm{L}$. Proteins were tested in triplicates and the fluorescence was monitored using a 96-well Real-Time PCR detection system (CFX96 qPCR machine, Bio-Rad), from $25^{\circ} \mathrm{C}$ to $95^{\circ} \mathrm{C}$, with a gradual temperature increase of $1^{\circ} \mathrm{C}$ every $10 \mathrm{~s}$.

\section{Protein thermostability assay}

LwaCas13a, HheCas13a and TccCas13a proteins were diluted to approximately $0.2 \mathrm{mg} / \mathrm{mL}$ in protein storage buffer (50 mM Tris-HCl pH 7.5, $600 \mathrm{mM} \mathrm{NaCl}, 5 \%$ glycerol, $2 \mathrm{mM}$ DTT) and incubated at a range of temperatures $\left(37^{\circ} \mathrm{C}, 60^{\circ} \mathrm{C}, 70^{\circ} \mathrm{C}\right.$, and $\left.90^{\circ} \mathrm{C}\right)$ for $30 \mathrm{~min}$. Samples were centrifuged in a microcentrifuge at $14,200 \mathrm{rpm}$ for $25 \mathrm{~min}$. A total of $5 \mathrm{~mL}$ of the supernatant was mixed with the same volume of protein 
sample loading buffer and heated at $95^{\circ} \mathrm{C}$ for $10 \mathrm{~min}$. The samples were allowed to cool down on ice for 3 min and run on a 10\% NuPAGE Bis-Tris polyacrylamide gel (NP0301BOX, ThermoFisher). Protein thermostability assays for HheCas13a and TccCas13a ribonucleoproteins (RNPs) was performed similarly after an initial incubation of the proteins with $1 \mathrm{mM}$ of their cognate crRNAs for $5 \mathrm{~min}$ at $37^{\circ} \mathrm{C}$ in order to assemble the RNP before subjecting them to a range of temperatures.

\section{In vitro cis cleavage assays}

HheCas13a and TccCas $13 a$ cleavage reactions were performed at $37^{\circ} \mathrm{C}$ and $60^{\circ} \mathrm{C}$ with synthetic, in vitrotranscribed RNA targets. Briefly, for both HheCas13a or TccCas13a cleavage assays, cleavage reactions were carried out in 20-mL reaction volume with $50 \mathrm{nM}$ of either Cas13a protein, $50 \mathrm{nM}$ of their cognate crRNAs, and $100 \mathrm{nM}$ of target RNA in $1 \mathrm{x}$ isothermal buffer $(20 \mathrm{mM}$ Tris- $\mathrm{HCl} \mathrm{pH} \mathrm{8.8,50} \mathrm{mM} \mathrm{KCl,} 10 \mathrm{mM}$ $\left(\mathrm{NH}_{4}\right)_{2} \mathrm{SO}_{4}, 2 \mathrm{mM} \mathrm{MgSO}_{4}, 0.1 \%$ Tween 20) supplemented with an additional $6 \mathrm{mM} \mathrm{MgSO}_{4}$ (final of $8 \mathrm{mM}$ $\mathrm{MgSO}_{4}$ ); the reactions were then incubated at the indicated temperatures for $1 \mathrm{~h}$ (no pre-assembly of Cas13a protein and crRNA to form RNP was performed). The samples were then boiled at $70^{\circ} \mathrm{C}$ for 3 min in 2X RNA Loading Dye (B0363S, NEB) and cooled down on ice for 3 min before loading onto a $6 \%$ polyacrylamide-urea denaturing gel. Electrophoresis was conducted for $45 \mathrm{~min}$ at $25 \mathrm{~W}$. The gel was stained with SYBR Gold Nucleic Acid Gel Stain (S11494, ThermoFisher) for 10 min, briefly washed with 1X Tris borate EDTA buffer and visualized using a Bio-Rad Molecular Imager Gel Doc system.

\section{Fluorescent ssRNA cleavage assays}

For reporter screening and other fluorescence-based assays, $50 \mathrm{nM}$ of HheCas13a or TccCas13a recombinant protein was incubated with $50 \mathrm{nM}$ of the respective crRNA, $250 \mathrm{nM}$ of ssRNA reporter (either poly $(\mathrm{A})$, poly $(\mathrm{U})$, or mixed sequence reporter) in $1 \mathrm{X}$ isothermal buffer $(20 \mathrm{mM}$ Tris- $\mathrm{HCl} \mathrm{pH} \mathrm{8.8,50} \mathrm{mM} \mathrm{KCl,}$ $10 \mathrm{mM}\left(\mathrm{NH}_{4}\right)_{2} \mathrm{SO}_{4}, 2 \mathrm{mM} \mathrm{MgSO}_{4}, 0.1 \%$ Tween 20) supplemented with an additional $6 \mathrm{mM} \mathrm{MgSO}_{4}$ (final of $\left.8 \mathrm{mM} \mathrm{MgSO}_{4}\right), 0.8 \mathrm{U} / \mu \mathrm{L}$ RNaseOUT (10777019, Invitrogen) and $2 \mu \mathrm{L}$ of (1-100 nM) target RNA in a 20$\mu \mathrm{L}$ reaction volume. These reactions were incubated in a 96-well plate (BioRad) at different temperatures for $1 \mathrm{~h}$ in a 96-well Real-Time PCR detection system (CFX96 qPCR machine, Bio-Rad), with fluorescence measurements taken every 2 min using the FAM channel.

\section{Two-pot detection reactions}

Reverse transcription and LAMP isothermal amplification of target nucleic acids were conducted using the previously reported RT-LAMP primers [22]. Reactions were performed using 1.6 mM FIP/BIP primers (with the T7 promoter sequence added to either the FIP or BIP primer), $0.2 \mathrm{mM} \mathrm{F3/B3}$ primers, and $0.4 \mathrm{mM}$ LF/LB primers in 1X Isothermal Amplification Buffer $(20 \mathrm{mM}$ Tris-HCl pH 8.8, $50 \mathrm{mM} \mathrm{KCl,} 10 \mathrm{mM}$ 
$\left(\mathrm{NH}_{4}\right)_{2} \mathrm{SO}_{4}, 2 \mathrm{mM} \mathrm{MgSO}_{4}, 0.1 \%$ Tween 20) (B0537, NEB), $1.4 \mathrm{mM}$ dNTPs, $8 \mathrm{U}$ of Bst 2.0 WarmStart DNA Polymerase (M0538, NEB), 7.5 U of WarmStart RTx Reverse Transcriptase (M0380, NEB) and $6 \mathrm{mM}$ $\mathrm{MgSO}_{4}(\mathrm{~B} 1003, \mathrm{NEB})$ in $25-\mu \mathrm{L}$ reactions containing $100 \mathrm{cp} / \mu \mathrm{L}$ of SARS-CoV-2 control standards. The reactions were incubated at $62^{\circ} \mathrm{C}$ for $40 \mathrm{~min}$ in a PCR machine (C1000 touch thermal cycler, BioRad).

For subsequent Cas13a-based detection, $50 \mathrm{nM}$ of recombinant HheCas13a or TccCas13a protein was incubated with $50 \mathrm{nM}$ of the respective crRNA, $250 \mathrm{nM}$ of ssRNA reporter (Poly(U) ssRNA reporter for HheCas13a or mix ssRNA reporter for TccCas13a), $0.8 \mathrm{U} / \mu \mathrm{L}$ RNaseOUT, $2 \mathrm{U} / \mu \mathrm{L}$ Hi-T7 RNA polymerase (M0658S, NEB), $1 \mathrm{mM} \mathrm{NTPs,} \mathrm{and} 2 \mathrm{~mL}$ of the RT-LAMP reaction product. Reactions were run in a 96-well plate (BioRad) at $55^{\circ} \mathrm{C}$ for $1 \mathrm{~h}$ in a 96-well Real-Time PCR detection system (CFX96 qPCR machine, BioRad), with fluorescence measurements taken every 2 min using the FAM channel.

\section{One-pot detection reactions}

For Bst DNA polymerase screening and other optimization reactions, reverse transcription and LAMP isothermal amplification of the target nucleic acids, coupled with T7-mediated in vitro transcription and Cas13-based detection of the amplified and in vitro-transcribed target RNA, were carried out in the same tube. Reactions were performed using RT-LAMP primers at a final concentration of $1.6 \mathrm{mM}$ for FIP/BIP primers (with the T7 promoter sequence added to either the FIP or BIP primer), $0.2 \mathrm{mM} \mathrm{F} 3 / \mathrm{B} 3$ primers, and $0.4 \mathrm{mM} \mathrm{LF} / \mathrm{LB}$ primers, in $1 \mathrm{X}$ Isothermal Amplification Buffer (from a different vendor from the Bst DNA polymerase screening reactions) or from Lucigen (30027, Lucigen) in other optimization experiments, $1.4 \mathrm{mM}$ dNTPs, $0.32 \mathrm{U} / \mu \mathrm{L}$ Bst DNA Polymerase (from a different vendor from the Bst DNA polymerase screening reactions) or from Lucigen (30027, Lucigen), $0.45 \mathrm{U} / \mu \mathrm{L}$ of WarmStart RTx Reverse Transcriptase (M0380, NEB), $6 \mathrm{mM} \mathrm{MgSO}_{4}, 0.8 \mathrm{U} / \mu \mathrm{L}$ RNaseOUT, $0.5 \mathrm{mM}$ NTPS, $2 \mathrm{U} / \mu \mathrm{L}$ Hi-T7 RNA polymerase (M0658S, NEB), $0.4 \mathrm{U} / \mu \mathrm{L}$ thermostable inorganic pyrophosphatase (M0296, NEB), $250 \mathrm{nM}$ RNA reporter, $50 \mathrm{nM}$ Cas13, $50 \mathrm{nM}$ crRNA, and $2 \mu \mathrm{L}$ of template RNA in 25- $\mu \mathrm{L}$ reactions. These reactions were incubated in a 96-well plate (BioRad) at $55^{\circ} \mathrm{C}$ (or as otherwise indicated) for $1-2 \mathrm{~h}$ in a 96 -well RealTime PCR detection system (CFX96 qPCR machine, Bio-Rad), with fluorescence measurements taken every 2 min using the FAM channel.

\section{OPTIMA-dx reaction}

The reaction was performed using RT-LAMP primers at a final concentration of $1.6 \mathrm{mM}$ FIP/BIP primers (with the T7 promoter sequence added to either the FIP or BIP primer), $0.2 \mathrm{mM} \mathrm{F3/B3}$ primers, and $0.4 \mathrm{mM}$ LF/LB primers, in 1X Isothermal Amplification Buffer from Lucigen (30027, Lucigen), $1.4 \mathrm{mM}$ dNTPs, Bst DNA Polymerase (30027, Lucigen), 2 U/ $\mu$ L SuperScript IV reverse transcriptase (18090010, 
Invitrogen), $6 \mathrm{mM} \mathrm{MgSO}_{4}, 0.1 \mathrm{U} / \mu \mathrm{L}$ thermostable RNAse $\mathrm{H}$ (M0523S, NEB), $0.8 \mathrm{U} / \mu \mathrm{L}$ RNasin plus (N2611, Promega), $0.5 \mathrm{mM}$ NTPs, $4 \mathrm{U} / \mu \mathrm{L} \mathrm{Hi}$-T7 RNA polymerase (M0658S, NEB), $0.4 \mathrm{U} / \mu \mathrm{L}$ thermostable inorganic pyrophosphatase (M0296, NEB), $750 \mathrm{nM}$ ssRNA HEX reporter, $50 \mathrm{nM}$ Cas13, $50 \mathrm{nM}$ crRNA, and $2 \mu \mathrm{L}$ of template RNA in $25-\mu \mathrm{L}$ reactions. A detailed protocol for the OPTIMA-dx reaction setup is provided in supplementary note 1.

\section{Development of OPTIMA-dx mobile app application}

See supplementary note 2 for detailed description.

\section{Declarations}

\section{Author information}

Corresponding Author

*E-mail: magdy.mahfouz@kaust.edu.sa

ORCID

Magdy Mahfouz: 0000-0002-0616-6365

\section{Notes}

The authors declare competing financial interests. A patent application has been filed based on this work.

\section{Author contributions}

M.M. conceived the research and analyzed data. A.M. conceived and designed the experiments. A.M., T.M., M.L.M., Q.W. and Z.A. performed the research and analyzed data. C.Z., R.A., and B.G. developed the OPTIMA-dx application. M.M. and A.M. wrote the paper with input from all authors.

\section{Acknowledgments}

We would like to thank Mr. Mohammad Alarawi for providing the RNA of SARS-CoV-2 clinical samples. We thank members of the genome engineering and synthetic biology laboratory for insightful discussions 
and technical support. This work was supported, in part, by the Smart Health Initiative at KAUST and the IAF and NTGC grants from the KAUST IED to MM.

\section{References}

1. Kilic, T., R. Weissleder, and H. Lee, Molecular and Immunological Diagnostic Tests of COVID-19: Current Status and Challenges. iScience, 2020. 23(8): p. 101406.

2. Barrangou, R. and L.A. Marraffini, CRISPR-Cas systems: Prokaryotes upgrade to adaptive immunity. Mol Cell, 2014. 54(2): p. 234-44.

3. Hsu, P.D., E.S. Lander, and F. Zhang, Development and applications of CRISPR-Cas9 for genome engineering. Cell, 2014. 157: p. 1262-1278.

4. Abudayyeh, 0.0., et al., C2c2 is a single-component programmable RNA-guided RNA-targeting CRISPR effector. Science, 2016. 353(6299): p. aaf5573.

5. Ackerman, C.M., et al., Massively multiplexed nucleic acid detection with Cas13. Nature, 2020. 582(7811): p. 277-282.

6. Barnes, K.G., et al., Deployable CRISPR-Cas13a diagnostic tools to detect and report Ebola and Lassa virus cases in real-time. Nature Communications, 2020. 11(1): p. 4131.

7. Gootenberg, J.S., et al., Nucleic acid detection with CRISPR-Cas13a/C2c2. Science, 2017.

8. Freije, C.A., et al., Programmable Inhibition and Detection of RNA Viruses Using Cas13. Mol Cell, 2019. 76(5): p. 826-837 e11.

9. Gootenberg, J.S., et al., Multiplexed and portable nucleic acid detection platform with Cas13, Cas12a, and Csm6. Science, 2018. 360(6387): p. 439-444.

10. Abudayyeh, O.O. and J.S. Gootenberg, CRISPR diagnostics. Science, 2021. 372(6545): p. 914-915.

11. Chertow, D.S., Next-generation diagnostics with CRISPR. Science, 2018. 360(6387): p. 381-382.

12. Zuo, X., C. Fan, and H.-Y. Chen, Biosensing: CRISPR-powered diagnostics. Nature Biomedical Engineering, 2017. 1(6): p. 0091.

13. Freije, C.A. and P.C. Sabeti, Detect and destroy: CRISPR-based technologies for the response against viruses. Cell Host Microbe, 2021. 29(5): p. 689-703.

14. McDermott, J.H., et al., The rise of point-of-care genetics: how the SARS-CoV-2 pandemic will accelerate adoption of genetic testing in the acute setting. Eur J Hum Genet, 2021. 
15. van Dongen, J.E., et al., Point-of-care CRISPR/Cas nucleic acid detection: Recent advances, challenges and opportunities. Biosens Bioelectron, 2020. 166: p. 112445.

16. Marsic, T., et al., Vigilant: An Engineered VirD2-Cas9 Complex for Lateral Flow Assay-Based Detection of SARS-CoV2. Nano Lett, 2021.

17. Broughton, J.P., et al., CRISPR-Cas12-based detection of SARS-CoV-2. Nat Biotechnol, 2020. 38(7): p. 870-874.

18. Agrawal, S., et al., Rapid detection of SARS-CoV-2 with Cas13. medRxiv, 2020.

19. Patchsung, M., et al., Clinical validation of a Cas13-based assay for the detection of SARS-CoV-2 RNA. Nat Biomed Eng, 2020. 4(12): p. 1140-1149.

20. Arizti-Sanz, J., et al., Streamlined inactivation, amplification, and Cas13-based detection of SARSCoV-2. Nat Commun, 2020. 11(1): p. 5921.

21. Thompson, D. and Y. Lei, Mini review: Recent progress in RT-LAMP enabled COVID-19 detection. Sensors and Actuators Reports, 2020. 2(1): p. 100017.

22. Joung, J., et al., Detection of SARS-COV-2 with SHERLOCK One-Pot Testing. N Engl J Med, 2020. 383(15): p. 1492-1494.

23. Mabey, D., et al., Diagnostics for the developing world. Nat Rev Microbiol, 2004. 2(3): p. 231-40.

24. Koeck, D.E., et al., Herbinix hemicellulosilytica gen. nov., sp. nov., a thermophilic cellulosedegrading bacterium isolated from a thermophilic biogas reactor. Int J Syst Evol Microbiol, 2015. 65(8): p. 2365-2371.

25. East-Seletsky, A., et al., RNA Targeting by Functionally Orthogonal Type VI-A CRISPR-Cas Enzymes. Mol Cell, 2017. 66(3): p. 373-383 e3.

26. Curtis, K.A., et al., A multiplexed RT-LAMP assay for detection of group M HIV-1 in plasma or whole blood. J Virol Methods, 2018. 255: p. 91-97.

27. Knott, G.J. and J.A. Doudna, CRISPR-Cas guides the future of genetic engineering. Science, 2018. 361(6405): p. 866-869.

28. Mahas, A., C. Neal Stewart, Jr., and M.M. Mahfouz, Harnessing CRISPR/Cas systems for programmable transcriptional and post-transcriptional regulation. Biotechnol Adv, 2017.

29. Abbott, T.R., et al., Development of CRISPR as an Antiviral Strategy to Combat SARS-CoV-2 and Influenza. Cell, 2020. 181(4): p. 865-876 e12. 
30. Lotfi, M. and N. Rezaei, CRISPR/Cas13: A potential therapeutic option of COVID-19. Biomed Pharmacother, 2020. 131: p. 110738.

31. Gao, J., et al., A New Tool for CRISPR-Cas13a-Based Cancer Gene Therapy. Mol Ther Oncolytics, 2020. 19: p. 79-92.

32. Palaz, F., et al., CRISPR-Cas13 System as a Promising and Versatile Tool for Cancer Diagnosis, Therapy, and Research. ACS Synth Biol, 2021.

33. Kontermann, R.E., Strategies for extended serum half-life of protein therapeutics. Curr Opin Biotechnol, 2011. 22(6): p. 868-76.

34. Abudayyeh, 0.0., et al., RNA targeting with CRISPR-Cas13. Nature, 2017. 550(7675): p. 280-284.

35. Harrington, L.B., et al., A thermostable Cas 9 with increased lifetime in human plasma. Nat Commun, 2017. 8(1): p. 1424.

36. Narasimhan, D., et al., Structural analysis of thermostabilizing mutations of cocaine esterase. Protein Eng Des Sel, 2010. 23(7): p. 537-47.

37. Mougiakos, I., et al., Characterizing a thermostable Cas9 for bacterial genome editing and silencing. Nat Commun, 2017. 8(1): p. 1647.

38. Ali, Z., et al., iSCAN: An RT-LAMP-coupled CRISPR-Cas12 module for rapid, sensitive detection of SARS-CoV-2. Virus Res, 2020. 288: p. 198129.

39. Li, L., et al., HOLMESV2: A CRISPR-Cas12b-Assisted Platform for Nucleic Acid Detection and DNA Methylation Quantitation. ACS Synth Biol, 2019. 8(10): p. 2228-2237.

40. Santiago-Frangos, A., et al., Intrinsic Signal Amplification by Type-III CRISPR-Cas Systems Provides a Sequence-Specific Viral Diagnostic. medRxiv, 2020: p. 2020.10.14.20212670.

41. Steens, J.A., et al., SCOPE: Flexible targeting and stringent CARF activation enables type III CRISPRCas diagnostics. bioRxiv, 2021: p. 2021.02.01.429135.

42. Couvin, D., et al., CRISPRCasFinder, an update of CRISRFinder, includes a portable version, enhanced performance and integrates search for Cas proteins. Nucleic Acids Res, 2018. 46(W1): p. W246W251.

43. Biswas, A., et al., CRISPRDetect: A flexible algorithm to define CRISPR arrays. BMC Genomics, 2016. 17: p. 356.

44. Kellner, M.J., et al., SHERLOCK: nucleic acid detection with CRISPR nucleases. Nat Protoc, 2019. 14(10): p. 2986-3012. 
A

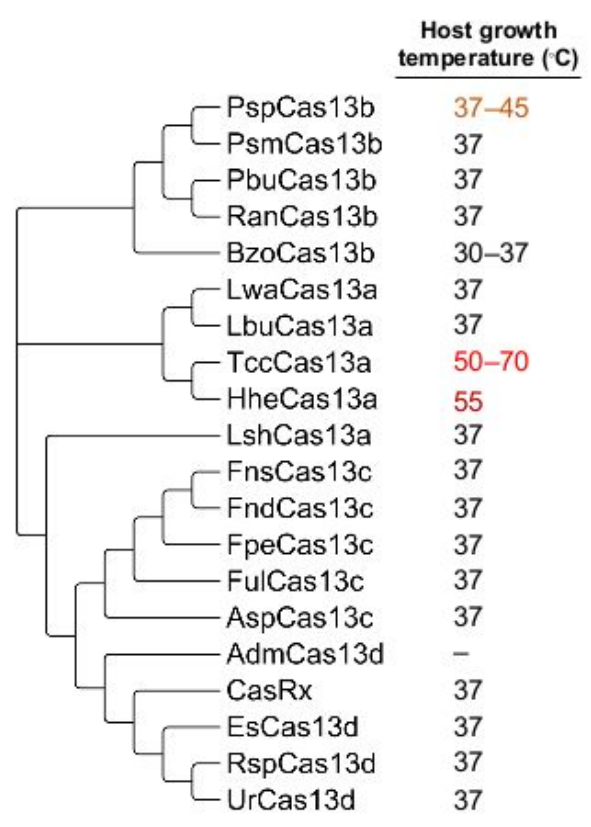

C

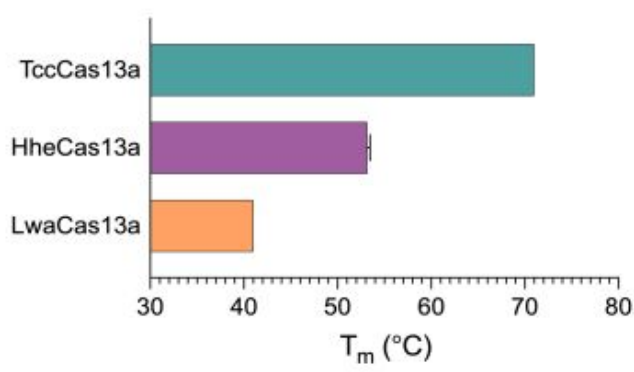

E

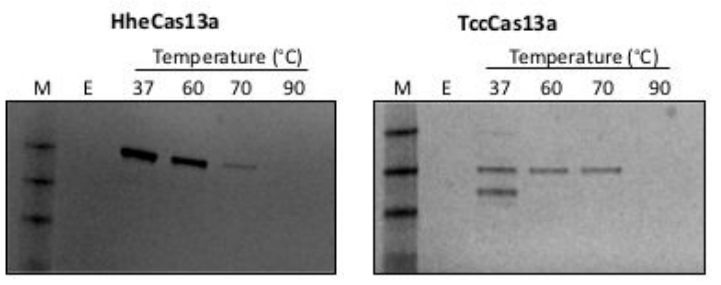

B

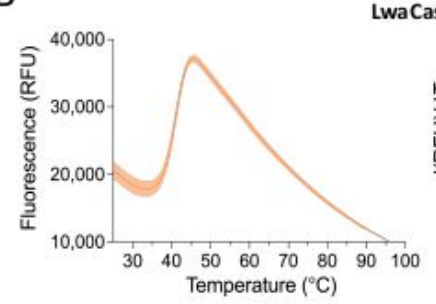

LwaCas13a

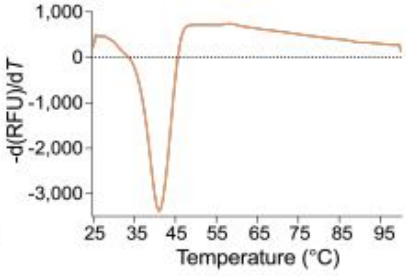

Hhe Cas13a
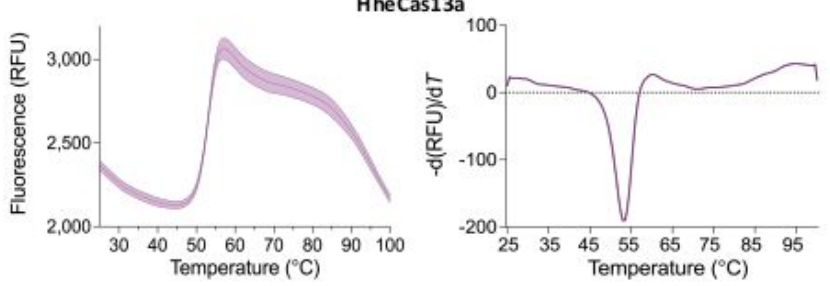

TccCas 13a
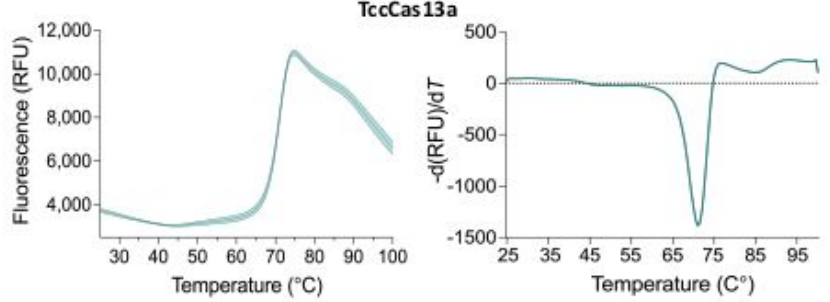

D
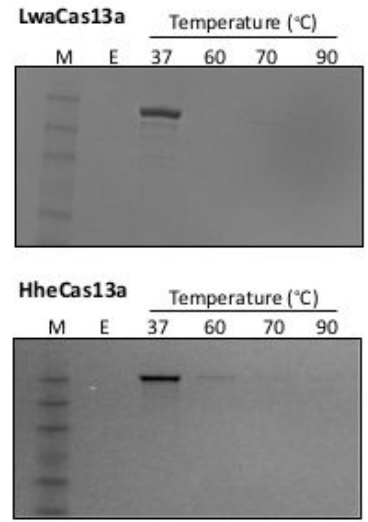

TccCas13a Temperature ${ }^{\circ} \mathrm{C}$

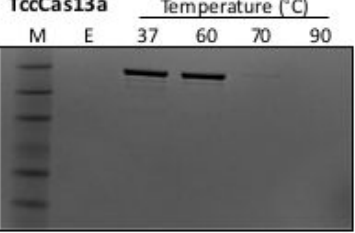

\section{Figure 1}

Thermostability analysis of thermophilic Cas13 effectors a Maximum-likelihood phylogenetic tree of Cas13 proteins from different organisms. The tree was generated using MEGA X software. Most selected proteins were isolated from mesophilic bacteria, although several have been cultivated as thermophiles, 
and thus offer an interesting collection of high-temperature stable proteins. TccCas13a and HheCas13a were selected as potentially thermophilic Cas13 proteins. b Differential scanning fluorimetry (DSF) profiles of protein melting point using a conventional real-time PCR instrument. The peak in the left graph indicates protein denaturation. The right-side graph is the derivative of the left-side graph. $c$ Denaturation temperature of TccCas13a, HheCas13a, and LwaCas13a proteins, as determined by DSF in Fig. 1b. Data are shown as mean \pm standard deviation (SD) $(n=3)$. d SDS-PAGE illustrating the protein stability of TccCas13a, HheCas13a, and LwaCas13a after incubation for 30 min at different temperatures. e SDSPAGE showing the protein stability of TccCas13a and HheCas13a ribonucleoproteins (RNPs) assembled with their cognate crRNAs. After assembly with crRNAs, TccCas13a and HheCas13a RNPs were incubated for $30 \mathrm{~min}$ at different temperatures before electrophoresis. 
A
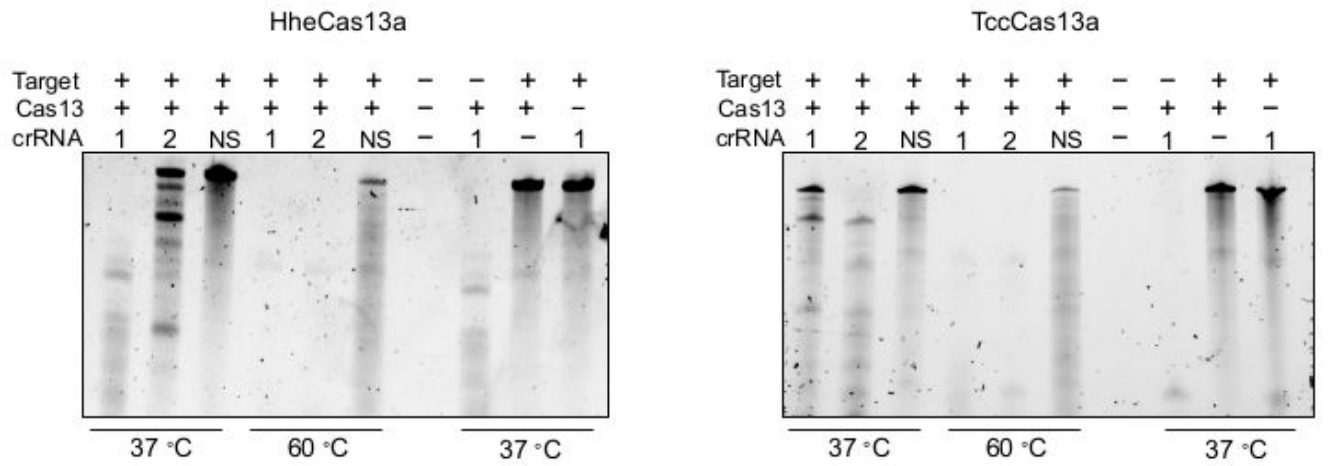

B

C
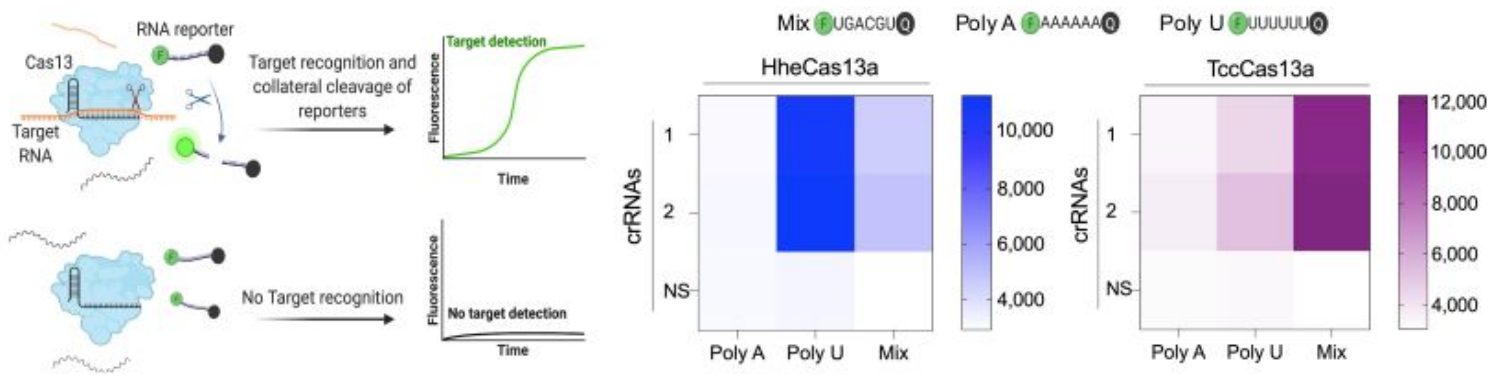

D

$\mathrm{E}$
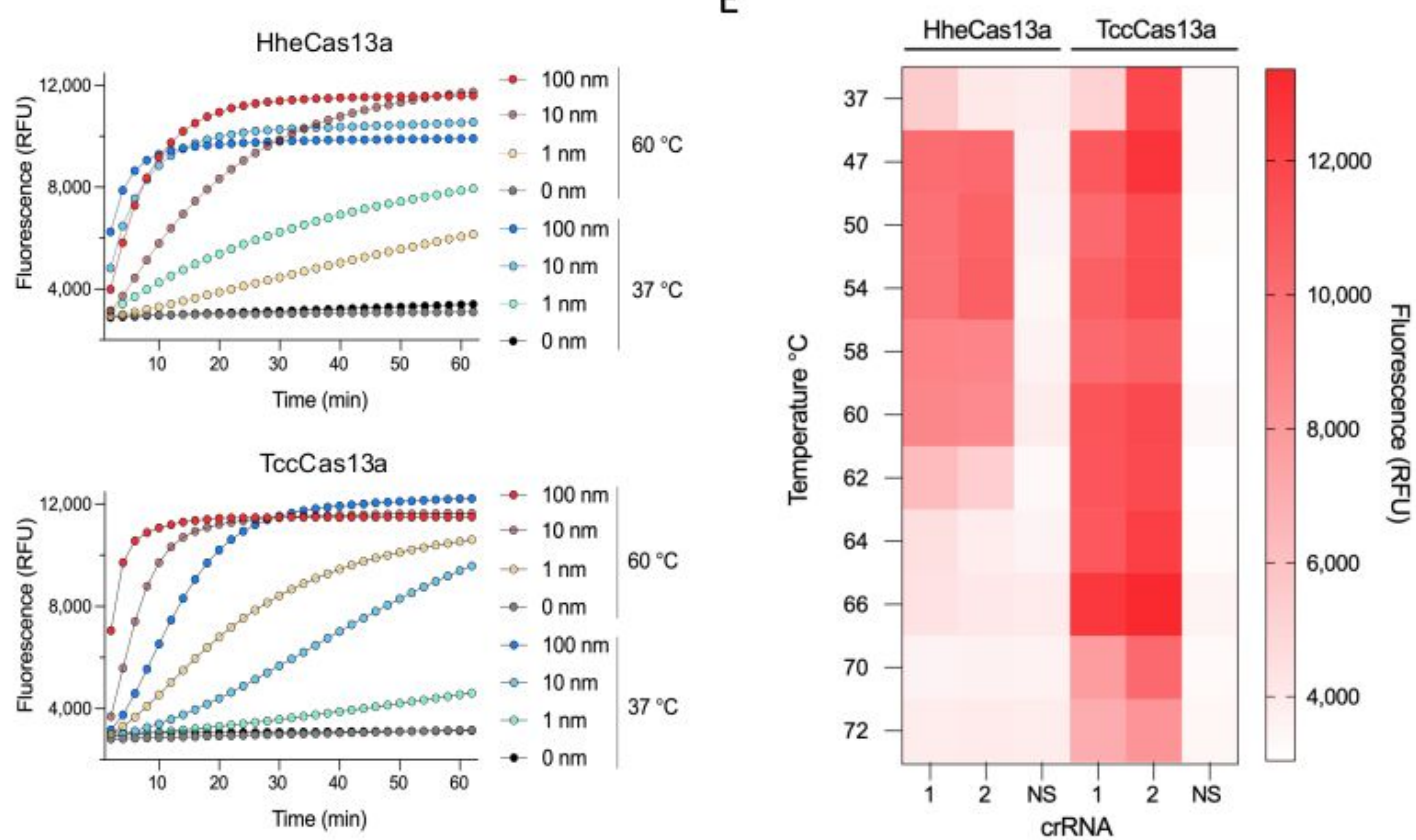

\section{Figure 2}

Characterization of cis and trans cleavage activities of thermophilic HheCas13a and TccCas13a. a Representative denaturing gel showing the targeted in vitro RNase cleavage activity of HheCas $13 \mathrm{a}$ and TccCas13a proteins when incubated with ssRNA target and different crRNAs at different temperatures. NS: non-specific crRNA control. b Schematic representation of specific Cas13-based detection. Specific target recognition by the Cas13 RNP triggers non-specific, collateral activity that cleaves the reporters, 
resulting in a detectable fluorescent signal. Each ssRNA reporter carries a fluorescent molecule and a fluorescence quencher. $\mathrm{c}$ HheCas13a and TccCas13a collateral cleavage preference for the ssRNA reporter. Reactions consisting of HheCas13a or TccCas13a and their respective cognate crRNAs or nonspecific crRNA (NS) control were performed in the presence of ssRNA target and one of three ssRNA reporters. NS: non-specific crRNA. Data are shown as mean $(n=3)$. ssRNA reporter sequences are shown on top of the panel. $d$ Measurement of real-time fluorescence output comparing the detection activity of HheCas13a and TccCas13a at three different target RNA concentrations $(100,10,1 \mathrm{nM})$ and two temperatures $\left(37^{\circ} \mathrm{C}\right.$ and $\left.60^{\circ} \mathrm{C}\right)$. Data are shown as mean $(n=3)$. e End-point activity of HheCas $13 \mathrm{a}$ and TccCas13a at different temperatures using their preferred reporter after $30 \mathrm{~min}$. Two crRNAs and a nonspecific crRNA were tested for each. 
A

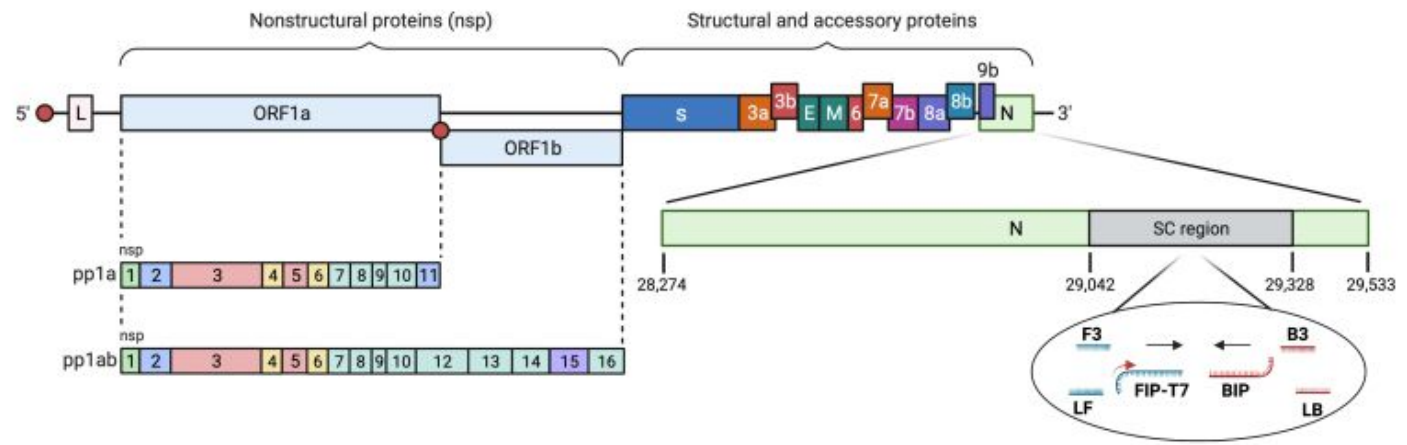

B

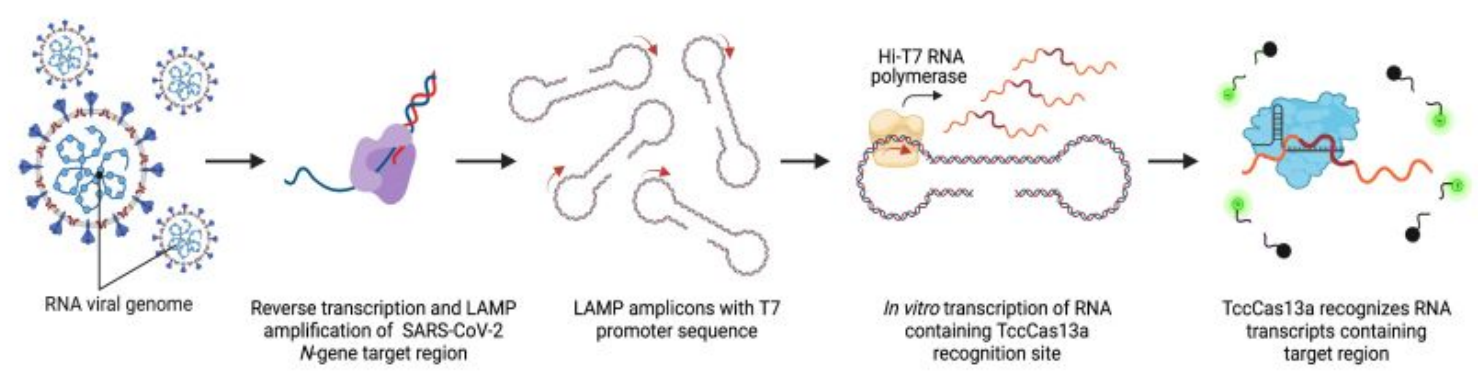

$\mathrm{C}$

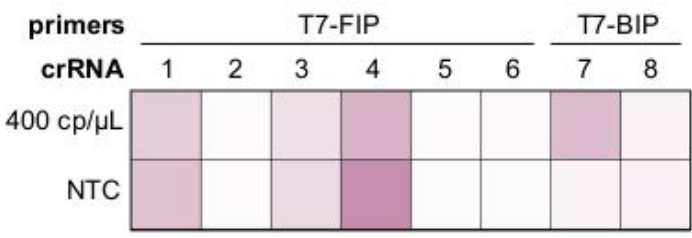

HheCas13a

D

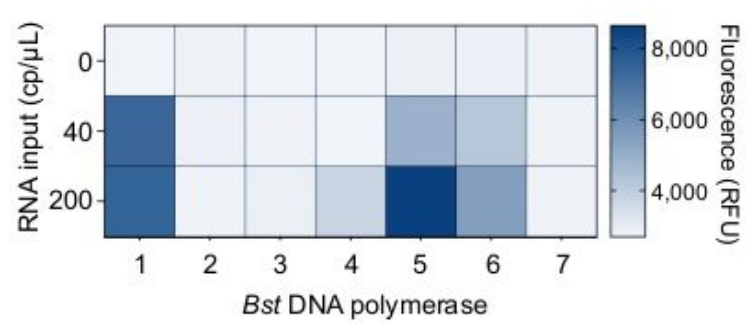

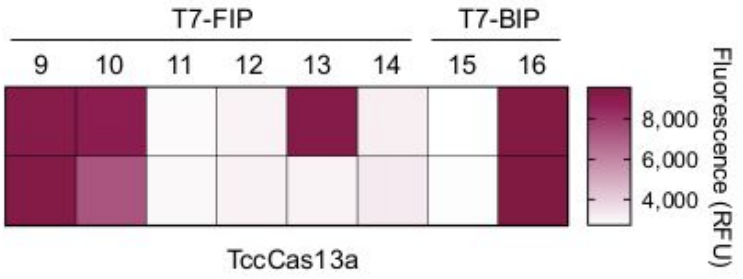

$\mathrm{E}$

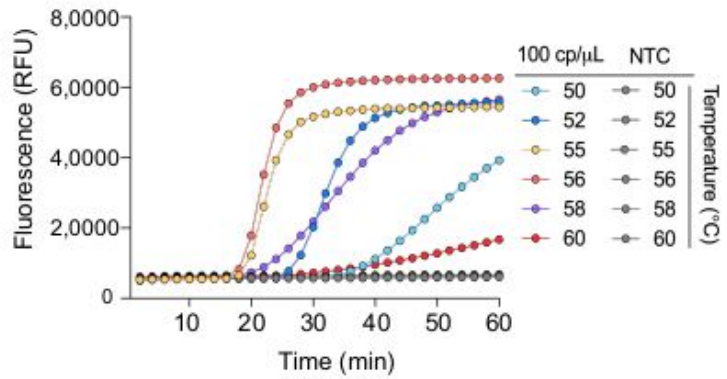

\section{Figure 3}

Establishment and optimization of one-pot SARS-CoV-2 detection using the thermophilic TccCas13a protein. a Schematic representation of the SARS-CoV-2 genome showing the region targeted by RT-LAMP amplification and the crRNA target sequence. The small red arrow on the T7-FIP primer indicates the T7 promoter sequence. b Overview of the assay workflow. The detection protocol consists of three distinct steps, all carried out in the same tube and at the same temperature $\left(56^{\circ} \mathrm{C}\right)$. Following the extraction of 
viral RNA, specific target sequences within the viral RNA are reverse-transcribed (RT) into cDNA and amplified with RT-LAMP isothermal amplification using LAMP primers containing the T7 promoter sequence (small red arrow on the T7-FIP primer). The resulting RT-LAMP amplicons are subsequently/simultaneously used for in vitro transcription using the thermostable Hi-T7 RNA polymerase, producing RNA transcripts that are recognized and targeted simultaneously by the thermophilic TccCas13a protein. Recognition of the RNA transcripts by TccCas13a triggers Cas 13 collateral cleavage activity, resulting in trans-cleavage of the reporter probe conjugated to the HEX or FAM fluorophores. c Trans cleavage activity of HheCas13a and TccCas13a using different crRNAs in one-pot reactions using SARS-CoV-2 genomic standards as input. The assay was performed as described in Material and Methods. End-point fluorescence signal detection was carried out after $80 \mathrm{~min}$. NTC: No template control. T7-FIP: modified RT-LAMP FIP primer with T7 promoter sequence. T7-BIP: modified RTLAMP BIP primer with T7 promoter sequence. Data are shown as mean $(n=3)$. d Activity screening of different commercially available Bst DNA polymerases for suitability in one-pot assays. 1- Bst DNA polymerase, exonuclease minus (Lucigen, 30027), 2- Bst DNA polymerase (web SCIENTIIFIC, S600), 3- Bst 2.0 WarmStart DNA polymerase (NEB, M0538), 4- Bst 3.0 DNA polymerase (NEB, M0374), 5- Bsm DNA polymerase, large fragment (ThermoFisher Scientific, EP0691), 6- Bst X DNA polymerase (enzymatics, P7390), 7- WarmStart LAMP kit (DNA\&RNA) (NEB, E1700). Data are shown as means $(n=3)$ and represent end-point fluorescence values after $80 \mathrm{~min}$. e Performance of one-pot detection assay at different temperatures, as determined by real-time fluorescence at a given target RNA concentration (100 $\mathrm{cp} / \mu \mathrm{L})$. The best performance was achieved at $56^{\circ} \mathrm{C}$. Data are shown as mean $(n=3)$. 
A

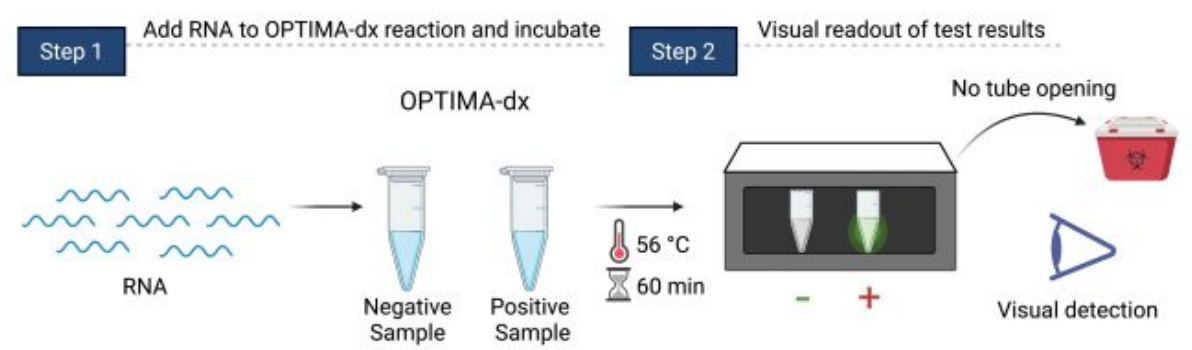

B

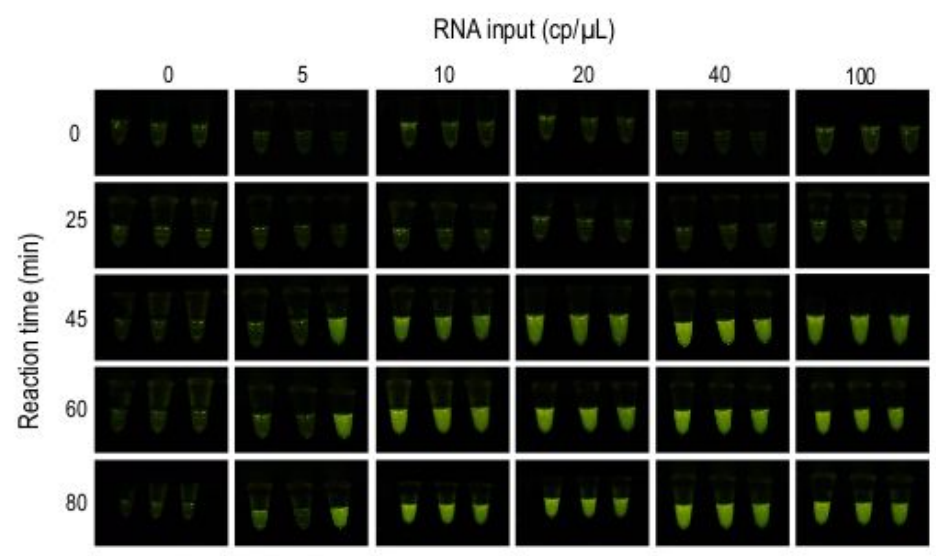

C

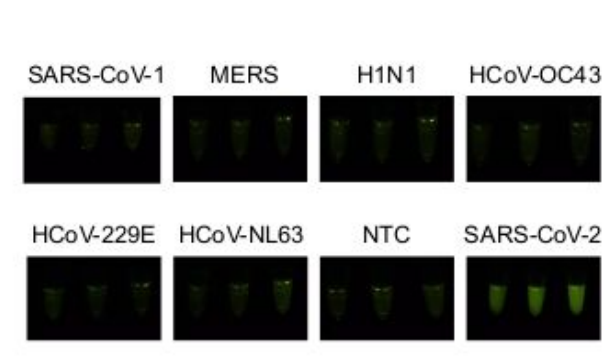

D

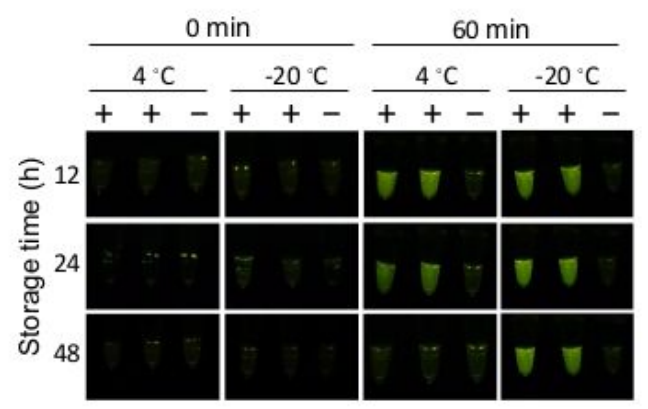

$\mathrm{E}$

$\mathrm{F}$
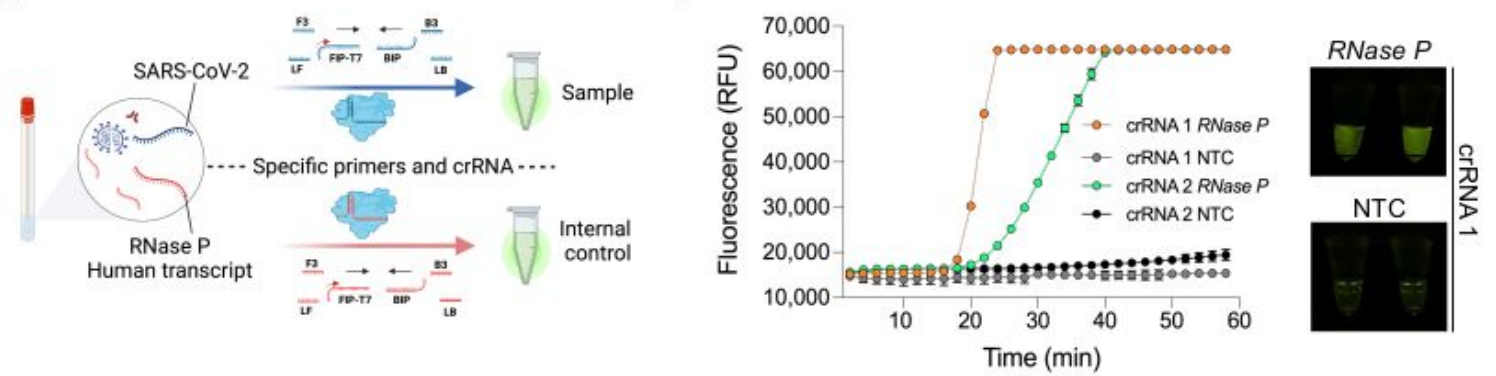

Figure 4

Evaluation of OPTIMA-dx for the detection of SARS-CoV-2. a Schematic representation of SARS-CoV-2 RNA detection in one-pot assays and visual detection using the P51 Molecular Fluorescence Viewer. As the test is performed in a single pot, there is no need to open the reaction tube so it can be discarded without opening, thus avoiding the possibility of contamination at the point of care site. b Assessment of the sensitivity of OPTIMA-dx and the effect of reaction incubation time on performance using 
fluorescence-based visual detection. Fluorescence rises above background after 45 min with little improvement as time increases. Three replicates were performed for each treatment. c Evaluation of specificity and cross-reactivity of OPTIMA-dx for SARS-CoV-2 RNA visual detection. Three replicates were performed for each treatment. NTC: No template control. d Effect of storage time of the OPTIMA-dx master mix at two temperatures on SARS-CoV-2 visual detection. Two replicates and one negative control (NTC: no template control) were tested for each treatment. e Schematic representation of dual detection of co-isolated and highly abundant human RNase P transcripts with OPTIMA-dx as an internal control for isolated RNA quality and integrity. $f$ Development and establishment of a human internal control for the OPTIMA-dx assay. Performance of the OPTIMA-dx assay with RNase P-specific LAMP-primers and two crRNAs, as measured by real-time fluorescence (left panel). Data are shown as means $\pm S D(n=3)$. The selected crRNA 1 was evaluated for visual detection (right panel) after 60 min incubation. NTC: no template control. 
A

$\begin{array}{lllllllllllllllllllll}\text { ID } & \text { A12 } & \text { B1 } & \text { B2 } & \text { D1 } & \text { E12 } & \text { F1 } & \text { F2 } & \text { F3 } & \text { F4 } & \text { F5 } & \text { F6 } & \text { F7 } & \text { F8 } & \text { F9 } & \text { F10 } & \text { F11 } & \text { F12 } & \text { G1 } & \text { G2 } & \text { G3 }\end{array}$

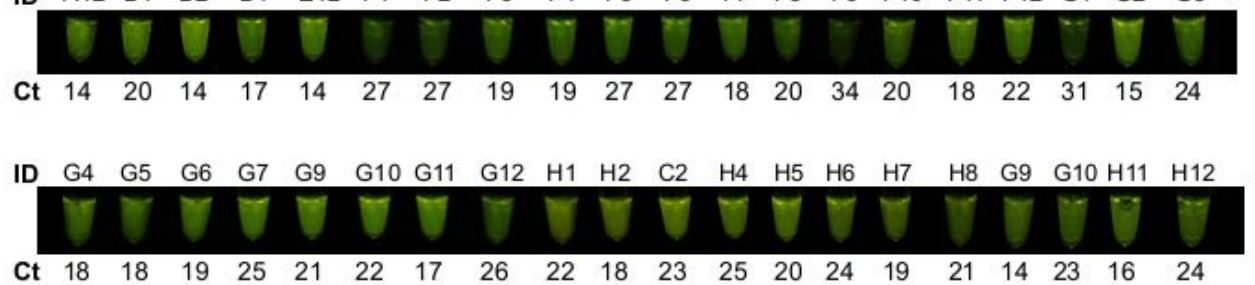

ID -ve 1 -ve 2 -ve 3 -ve 4 -ve 5 NTC

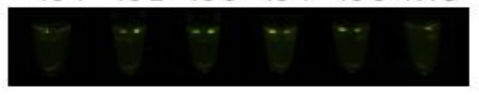

B
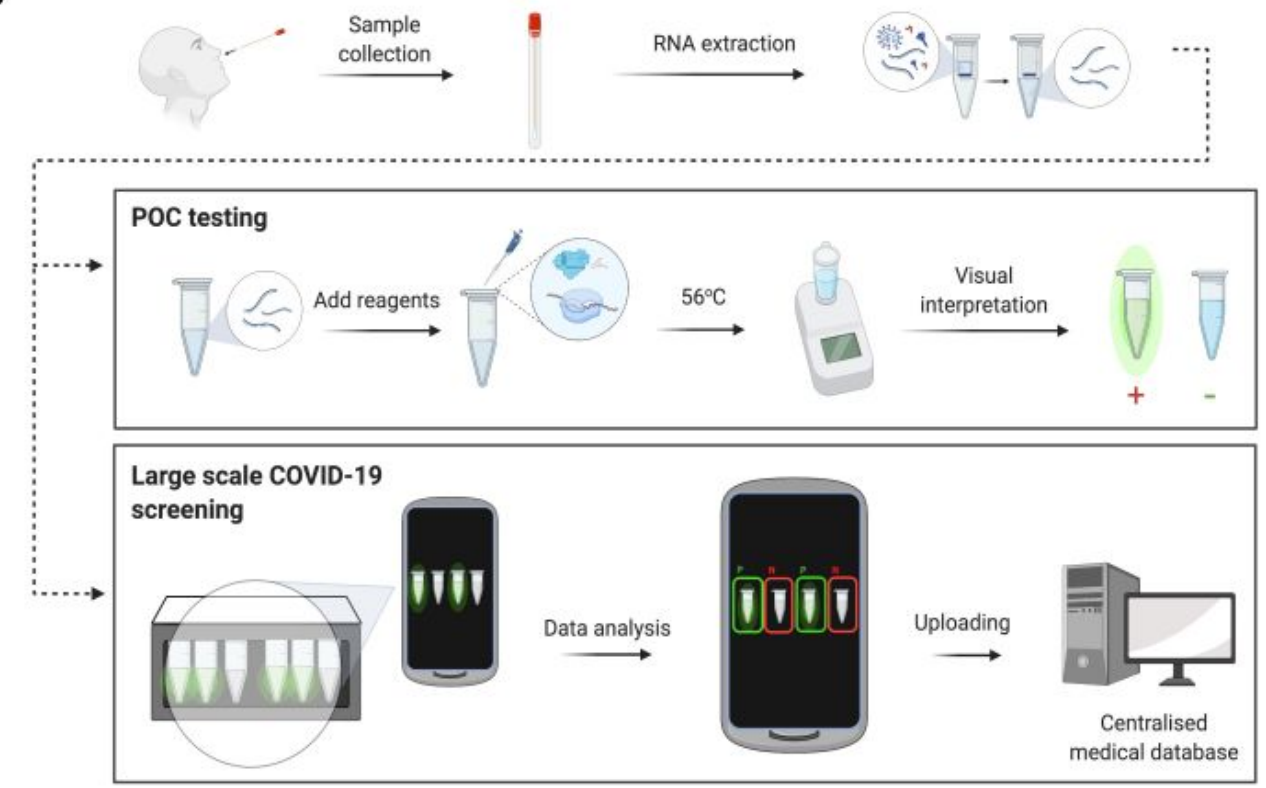

C

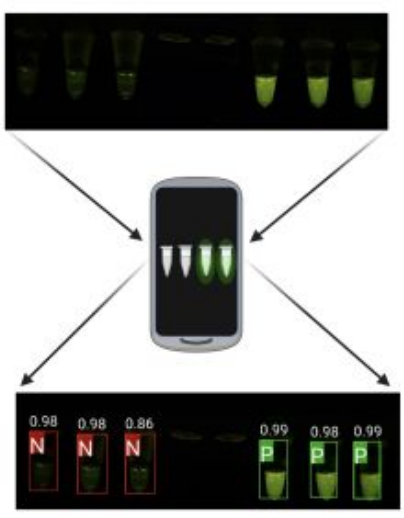

D

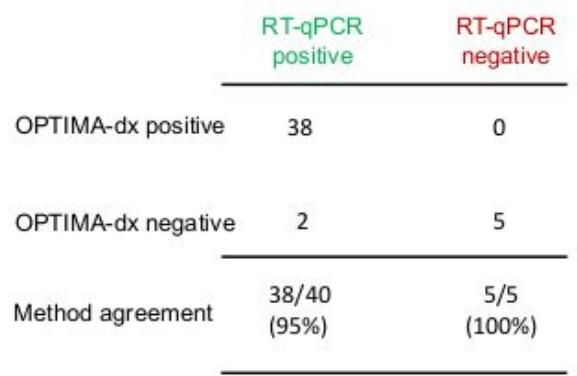

\section{Figure 5}

Validation of OPTIMA-dx performance on patient samples with the use of smartphones for SARS-CoV-2 detection. a Visual detection of SARS-CoV-2 RNA from samples collected from 45 patients by OPTIMA-dx after $1 \mathrm{~h}$. RT-qPCR Ct values are shown below each sample. b Overview of OPTIMA-dx workflow with mobile phone application for interpreting the OPTIMA-dx fluorescence readouts. The workflow shows the OPTIMA-dx protocol using RNA extracted from patient sample that is added to the preassembled one-pot 
reaction. The reaction is incubated at $56^{\circ} \mathrm{C}$ for $1 \mathrm{~h}$. To interpret the results, the OPTIMA-dx fluorescence readouts are visualized using p51 Molecular Fluorescence Viewer, and the results can be captured using mobile phone camera. The captured picture of the fluorescence readouts is processed with the app and interpreted as positive (P, green) or negative $(\mathrm{N}$, red). The OPTIMA-dx results can be uploaded to or shared with a centralised database. c Representative data showing the OPTIMA-dx results before (top panel) and after (lower panel) analysis with the smartphone application. $d$ Concordance between OPTIMA-dx results using the smartphone application and RT-qPCR detection on the 45 patient samples. See supplementary figure 7 and supplementary figure 8 for results with the smartphone application.

\section{Supplementary Files}

This is a list of supplementary files associated with this preprint. Click to download.

- SupplementaryInformationThermoCas13V3June7.pdf 